Document downloaded from:

http://hdl.handle.net/10251/121432

This paper must be cited as:

Company Rossi, R.; Egorova, V.; Jódar Sánchez, LA. (2018). Conditional full stability of positivity-preserving finite difference scheme for diffusion advection-reaction models. Journal of Computational and Applied Mathematics. 341:157-168.

https://doi.org/10.1016/j.cam.2018.02.031

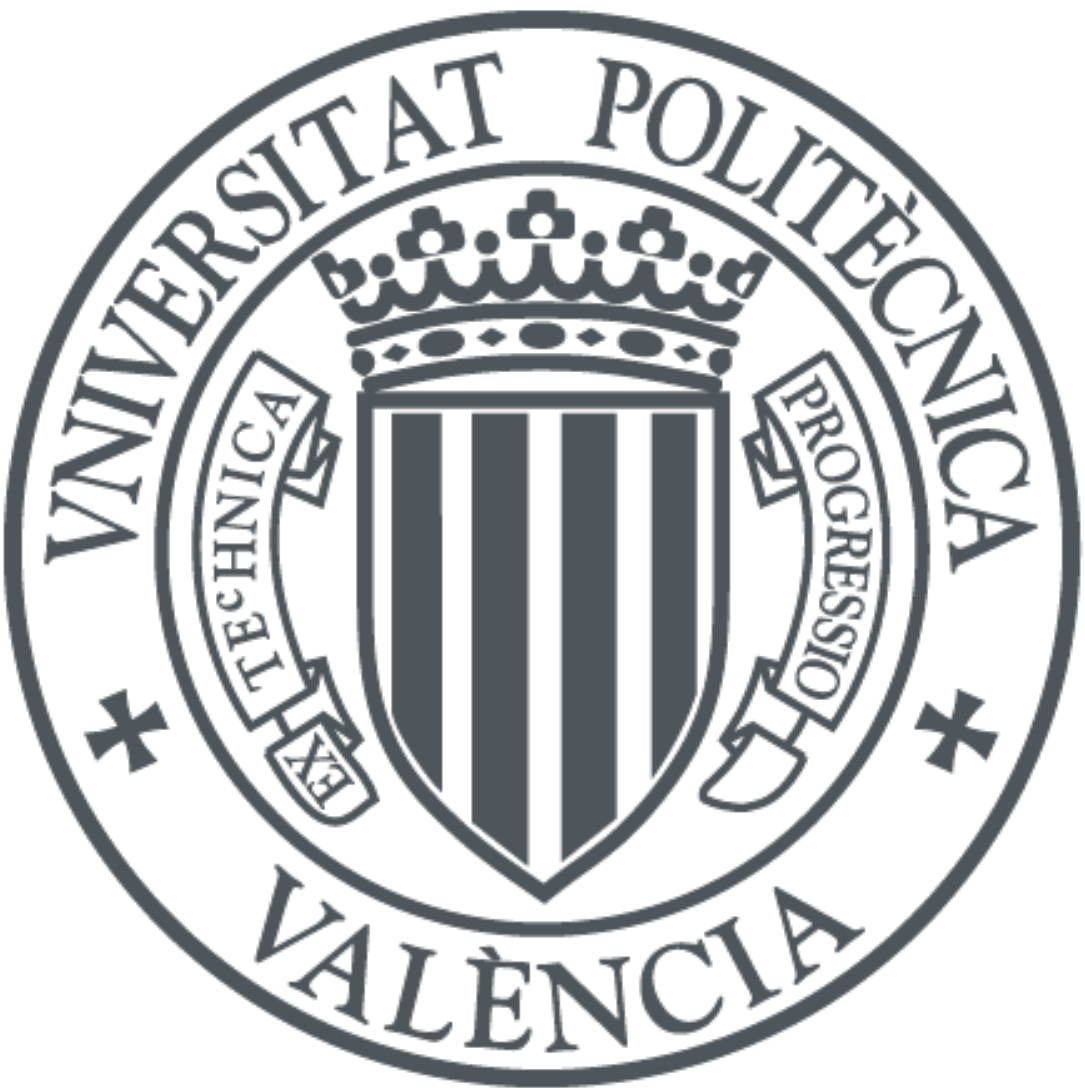

The final publication is available at

http://doi.org/10.1016/j.cam.2018.02.031

Copyright Elsevier

Additional Information 


\title{
Conditional full stability of positivity-preserving finite difference scheme for diffusion-advection-reaction models
}

\author{
Rafael Company ${ }^{1}$, Vera N. Egorova ${ }^{2}$, Lucas Jódar ${ }^{1}$ \\ ${ }^{1}$ Universitat Politécnica de València, camino de Vera s/n, 46022, Valencia, Spain \\ ${ }^{2}$ BCAM - Basque Center for Applied Mathematics, Al. de Mazarredo 14, 48009 Bilbao, \\ Basque Country, Spain
}

\begin{abstract}
The matter of the stability for multidimensional diffusion-advection-reaction problems treated with the semi-discretization method is remaining challenge because when all the stepsizes tend simultaneously to zero the involved size of the problem grows without bounds. Solution of such problems is constructed by starting with a semi-discretization approach followed by a full discretization using exponential time differencing and matrix quadrature rules. Analysis of the time variation of the numerical solution with respect to previous time level together with the use of logarithmic norm of matrices are the basis of the stability result. Sufficient stability conditions on stepsizes, that also guarantee positivity and boundedness of the solution, are found. Numerical examples in different fields prove its competitiveness with other relevant methods.
\end{abstract}

Keywords: Diffusion-advection-reaction, semi-discretization, exponential time differencing, finite difference, numerical analysis.

2010 MSC: 65M06, 65M12, 65M20

\section{Introduction}

Time-dependent diffusion-advection-reaction (DAR) models have application in a wide class of problems [1] appearing in many fields as fluid dynamics [1], biology [2], population dynamics [3], and financial mathematics [4], etc. ${ }_{5}$ Such time evolution problems are modeled by involving three factors: diffusion, advection and reaction. Diffusion deals with the dispersion given in the species involved in the process throughout the domain of the problem. Advection is 
related to the movement of species due to the fluid transport medium and the reaction explains the interaction through which the species are generated or 10 consumed [5].

The partial differential equation (PDE) that governs the diffusion-advectionreaction type processes adds significant mathematical complexity to be considered carefully when solving the problem. Well-posedness conditions for nonlinear second order PDEs problems including DAR initial value problems are given

15 in Section 4.9 of [6]. The notion of viscosity solutions provides a framework in which existence and uniqueness theorems may be proved by very efficient arguments. A rigorous study can be found in [7] for initial boundary second order PDEs.

It is important to point out that existence and uniqueness conditions uses to be much more restrictive that they appear frequently in practice. Even when such conditions are not fulfilled, the search of numerical solutions is important because the practitioners may have empirical evidences of the problem to check the reliability of the approximation.

Only in some particular cases it is possible to solve the DAR equations 25 exactly [8]. In a more general situation, numerical techniques are required. One of the common methods is a semi-discretization with respect to spatial variables achieving a system of ordinary differential equations (ODE). It is a fertile approach allowing two further alternatives. For the numerical solution of the systems of ODEs there are many numerical methods available, such as

30 Runge-Kutta methods [9] or the further time discretization deriving many types of finite difference schemes $[1,10,11]$.

Alternative approach, that it is used in the present study, is the exact integration of the semi-discretized equations using the Exponential Time Differencing (ETD) method [12] involving an integral term that needs to be approximated 35 because is expressed in terms of the unknown solution of the semi-discretized system of ODEs, that it has been recently treated in [13]. This approach has to afford the challenge of the computation of the inverse of matrices not always well conditioned when eigenvalues are close to zero [14]. 
Dealing with numerical finite difference methods, as the best model may 40 be wasted with careless analysis, it is convenient to study the stability of the numerical solution as all the stepsize tends to zero. However, as the spatial stepsize tends to zero the dimension of the matrix of the semi-discretized system of ODEs grows without end becoming a mathematical challenge. Apart from the stability, as the solutions of the problems represent concentrations, populations

45 or prices, the positivity is a necessary requirement for many practical problems.

In this paper we consider multidimensional heterogeneous DAR problems of the type

$$
\frac{\partial u}{\partial t}=\sum_{i=1}^{M} D_{i i}(\mathbf{x}) \frac{\partial^{2} u}{\partial x_{i}^{2}}+\sum_{i=1}^{M} \alpha_{i}(\mathbf{x}) \frac{\partial u}{\partial x_{i}}+F(\mathbf{x}, u),
$$

where $(\mathbf{x}, t) \in \Omega \times(0, T) \subset \mathbb{R}^{M+1}$, together with the non-negative initial and boundary conditions

$$
u(\mathbf{x}, 0)=U_{0}(\mathbf{x}) \leq 1, \mathbf{x} \in \Omega, \quad u(\mathbf{x}, t)=\phi(\mathbf{x}, t) \leq 1, \mathbf{x} \in \partial \Omega,
$$

where diffusion and advection coefficients $D_{i i}(\mathbf{x})$ and $\alpha_{i}(\mathbf{x})$ are continuous functions for $1 \leq i \leq M$ and $D_{i i}(\mathbf{x})>0$. The source term $F(\mathbf{x}, u)$ is positive admitting bounded partial derivatives $\left|\frac{\partial F}{\partial u}(\mathbf{x}, u)\right| \leq \lambda, 0 \leq u \leq 1$, and $F(\mathbf{x}, 1)=0$, $x \in \Omega$.

In this paper a semi-discretization method to solve (1)-(2) is presented together with a ETD scheme for the integration of the resulting system of ODEs using the accurate Simpson's rule and avoiding the calculation of the inverse of the coefficient matrix of the system. Furthermore, taking advantage of logarithmic norm of matrices and found properties of matrix exponential of the coefficient matrix a stability analysis is performed to guarantee conditionally

60 the boundedness of the solution independently of the size of the semi-discrete system.

This paper is organized as follows. Section 2 addresses the spatial semidiscretization and further proposed ETD scheme construction. Positivity and stability analysis is included into Section 3. In Section 4, a couple of numer- 
ical examples dealing with Fisher-Kolmogorov-Petrovsky-Piscounov equation

in Population Dynamics and Multi-asset American basket option in Financial Mathematics are included in order to illustrate the use of the proposed scheme.

\section{Semi-discretization and ETD scheme}

The numerical solution is constructed in the computational domain with limits $x_{i_{\min }}$ and $x_{i_{\max }}, i=1, \ldots, M$. A uniform mesh in each coordinate spatial computational grid of $N_{i}+1$ nodes with stepsizes $h_{i}$ takes the following form

$$
\xi_{i}^{j}=x_{i_{\min }}+j h_{i}, \quad h_{i}=\frac{x_{i_{\max }}-x_{i_{\min }}}{N_{i}}, 0 \leq j \leq N_{i}, 1 \leq i \leq M .
$$

An approximate solution at the point $\left(\boldsymbol{\xi}^{j}, t\right)=\left(\xi_{1}^{j_{1}}, \xi_{2}^{j_{2}}, \ldots, \xi_{M}^{j_{M}}, t\right)$ is denoted by $u_{j_{1}, \ldots, j_{M}}=u_{j_{1}, \ldots, j_{M}}(t)$. Let us denote the set of all mesh points by $\Gamma$, the subset of the mesh points located at the boundary of the numerical domain by

$$
\partial \Gamma=\left\{\left(\xi_{1}^{j_{1}}, \xi_{2}^{j_{2}}, \ldots, \xi_{M}^{j_{M}}\right) \mid \exists m, 1 \leq m \leq M, j_{m}=0 \text { or } j_{m}=N_{m}\right\},
$$

and the subset of interior nodes by $\stackrel{\circ}{\Gamma}=\Gamma \backslash \partial \Gamma$. Then semi-discretization of the equation (1) is obtained by using the second order central difference approximation for the spatial derivatives, resulting in the system of ODEs of the form

$$
\begin{aligned}
\frac{d u_{j_{1}, \ldots, j_{M}}}{d t} & =\sum_{i=1}^{M} D_{i i}\left(\boldsymbol{\xi}^{j}\right) \frac{u_{j_{1}, \ldots, j_{i}-1, \ldots, j_{M}}-2 u_{j_{1}, \ldots, j_{i}, \ldots, j_{M}}+u_{j_{1}, \ldots, j_{i}+1, \ldots, j_{M}}}{h_{i}^{2}} \\
& +\sum_{i=1}^{M} \alpha_{i}\left(\boldsymbol{\xi}^{j}\right) \frac{u_{j_{1}, \ldots, j_{i}+1, \ldots, j_{M}}-u_{j_{1}, \ldots, j_{i}-1, \ldots, j_{M}}}{2 h_{i}}+F\left(\boldsymbol{\xi}^{j}, u_{j_{1}, \ldots, j_{M}}\right) .
\end{aligned}
$$

Let us introduce the following notation for $i=1, \ldots, M$ :

$$
\begin{aligned}
h_{i} & =\beta_{i} h, \quad D\left(\boldsymbol{\xi}^{j}\right)=\sum_{i=1}^{M} \frac{D_{i i}\left(\boldsymbol{\xi}^{j}\right)}{\beta_{i}^{2}} \\
a_{0}^{j} & =-\frac{2}{h^{2}} D\left(\boldsymbol{\xi}^{j}\right), \\
a_{ \pm i}^{j} & =\frac{1}{h^{2}}\left(\frac{D_{i i}\left(\boldsymbol{\xi}^{j}\right)}{\beta_{i}^{2}} \pm \frac{h}{2 \beta_{i}} \alpha_{i}\left(\boldsymbol{\xi}^{j}\right)\right) .
\end{aligned}
$$


Let us denote by $\mathbf{u}=\mathbf{u}(t) \in \mathbb{R}^{N+1}$ the vector of all values $u_{j_{1}, \ldots, j_{M}}$, such that $\mathbf{u}=\left[u_{0}, \ldots, u_{N}\right]^{T}$, where $N+1$ means the total number of mesh points and each index $j, 0 \leq j \leq N$, has a one to one correspondence with the set of indexes $\left[j_{1}, \ldots, j_{M}\right]$ as follows

$$
\begin{gathered}
N+1=\prod_{i=1}^{M}\left(N_{i}+1\right)=\frac{1}{h^{M}} \prod_{i=1}^{M} \frac{x_{i_{\max }}-x_{i_{\min }}+\beta_{i} h}{\beta_{i}}, \\
{\left[j_{1}, \ldots, j_{M}\right] \equiv j=j_{1}+\sum_{m=2}^{M}\left(\prod_{n=1}^{m-1}\left(N_{n}+1\right)\right) j_{m} .}
\end{gathered}
$$

System (5) with the boundary and initial conditions (2) can be presented in the following vector form

$$
\frac{d \mathbf{u}}{d t}(t)=A \mathbf{u}(t)+\boldsymbol{f}(\boldsymbol{\xi}, \mathbf{u}) ; \quad \mathbf{u}(0)=\left[u_{0}(0), \ldots, u_{N}(0)\right]^{T},
$$

where $\boldsymbol{f}(\boldsymbol{\xi}, \mathbf{u})=\left[f_{0}, \ldots, f_{N}\right]^{T}, f_{j}=F\left(\boldsymbol{\xi}^{j}, \mathbf{u}\right)$, if $\boldsymbol{\xi}^{j} \in \stackrel{\circ}{\Gamma}$ and $f_{j}=\frac{\partial \phi\left(\boldsymbol{\xi}^{j}, t\right)}{\partial t}$ otherwise, $u_{j}(0)=U_{0}\left(\boldsymbol{\xi}^{j}\right), j=0, \ldots, N$.

Matrix $A$ is a sparse banded $(N+1) \times(N+1)$ matrix whose size depends on stepsize $h$ (see eq. (9)), and rows are entirely with zeros or containing $2 M+1$ non-zero entries. In fact, $A=\left(a_{i j}\right)_{0 \leq i, j \leq N}$, with only non-zero entries for $\boldsymbol{\xi}^{i} \in \stackrel{\circ}{\Gamma}$, such that

$$
a_{i i}=a_{0}^{i}, \quad a_{i, i \pm 1}=a_{ \pm 1}^{i}, \quad a_{i, i \pm \prod_{n=1}^{m-1}\left(N_{n}+1\right)}=a_{ \pm m}^{i}, 2 \leq m \leq M .
$$

Numerical solution of the system (11) is constructed by the ETD method [12]. Let us introduce temporal discretization with the fixed constant time step $k=\frac{T}{N_{t}}$, so $t^{n}=n k, n=0, \ldots, N_{t}-1$. Then the exact solution of the system of ODE (11) in some given interval $t \in\left[t^{n}, t^{n+1}\right]$ is given by Section 2.1 of [12]:

$$
\mathbf{u}\left(t^{n+1}\right)=e^{A k} \mathbf{u}\left(t^{n}\right)+\int_{0}^{k} e^{A s} \boldsymbol{f}\left(\boldsymbol{\xi}, \mathbf{u}\left(t^{n+1}-s\right)\right) d s .
$$

We propose a first explicit approximation of the integral in (13) by replacing $\mathbf{u}\left(t^{n+1}-s\right)$ by the known value $\mathbf{u}\left(t^{n}\right)$ corresponding to $s=k$. Let us denote $\mathbf{v}^{n+1}$ by

$$
\mathbf{v}^{n+1}=e^{A k} \mathbf{u}\left(t^{n}\right)+\left(\int_{0}^{k} e^{A s} d s\right) \boldsymbol{f}\left(\boldsymbol{\xi}, \mathbf{u}\left(t^{n}\right)\right)
$$


then in accordance with Section 2.1 of [12], the local truncation error is $O\left(k^{2}\right)$.

Instead of solving the integral $\int_{0}^{k} e^{A s} d s$ in exact form involving $A^{-1}$ like [15], as matrix $A$ can be singular or ill-conditioned, we use the accurate Simpson's rule, see [16],

$$
\int_{0}^{k} e^{A s} d s=k \varphi(A, k)+O\left(k^{5}\right), \quad \varphi(A, k)=\frac{1}{6}\left(I+4 e^{A \frac{k}{2}}+e^{A k}\right) .
$$

Let $\mathbf{u}^{n} \approx \mathbf{u}\left(t^{n}\right)$ be the numerical solution of the proposed fully discretized explicit scheme

$$
\mathbf{u}^{n+1}=e^{A k} \mathbf{u}^{n}+k \varphi(A, k) \boldsymbol{f}\left(\boldsymbol{\xi}, \mathbf{u}^{n}\right), \quad t^{n}=n k, n=0, \ldots, N_{t}-1 .
$$

According to (15) the local truncation error of the full discretized explicit 75 scheme (16) versus the ODE system (11) is of the second order in time.

\section{Positivity and stability}

In this section we pay attention to the stability in the infinity norm of the scheme (16) in the classical sense, i.e. that the numerical solution $\mathbf{u}^{n}$ remains bounded at each time level $n$, such that, $\left\|\mathbf{u}^{n}\right\|_{\infty} \leq C, 0 \leq n \leq N_{t}$, where $C_{80}$ is independent of the stepsizes $h$ and $k$. For the proposed scheme the stability analysis is a challenging task, because the dimension of the matrix $A$ grows as stepsizes decrease (see (3)) and the entries of the matrix $A$ also grows (see (8)).

For the sake of clarity in the presentation we recall some definitions and results that might be found in [17].

A matrix $A \in \mathbb{R}^{n \times n}$ is called the Metzler matrix if its off-diagonal entries are non-negative. If $A$ is the Metzler matrix, then $e^{A t} \geq 0$ for $t \geq 0$. It is well known the boundedness of exponential matrix norm by the exponential of the logarithmic norm $\mu[A]$, [18]:

$$
\left\|e^{A k}\right\| \leq e^{k \mu[A]} .
$$

Denoting the real part of complex number $x$ by $\Re(x), \mu_{\infty}[A]$ can be calcu- 
lated as follows, see [19], p. 33,

$$
\mu_{\infty}[A]=\max _{i}\left(\Re\left(a_{i i}\right)+\sum_{j \neq i}\left|a_{i j}\right|\right) .
$$

From (8) coefficients $a_{ \pm i}^{j}, i=1, \ldots, M$, are non-negative, and correspondingly matrix $A$ is Metzler, if stepsize $h$ is chosen as

$$
h \leq \min _{1 \leq i \leq M} \frac{2 D_{i i}(\boldsymbol{x})}{\beta_{i}\left|\alpha_{i}(\boldsymbol{x})\right|}, \quad \boldsymbol{x} \in \Omega .
$$

From (8), (12) and (18), one gets $\mu_{\infty}[A]=0$.

Thus, from (17) $\left\|e^{A k}\right\|_{\infty} \leq e^{0}=1$. And from (15), $\|\varphi(A, k)\|_{\infty} \leq 1$. $A$ has several zero rows, and their corresponding rows in $e^{A k}$ have only one entry equal to 1 while the other entries are zeros, then

$$
\left\|e^{A k}\right\|_{\infty}=\|\varphi(A, k)\|_{\infty}=1
$$

The non-negativity of $\boldsymbol{u}^{n}$ follows from the property of the Metzler matrix $A$, that is the exponential $e^{A k}$ is non-negative, and non-negativity of the source term and $\varphi(A, k)$. The boundedness of the solution $\left(u_{i}^{n} \leq 1,0 \leq i \leq N\right.$, $0 \leq n \leq N_{t}$ ) is proven by using the induction principle. Let us represent (16) as a function $g_{i}$ on the arguments $u_{0}^{n}, \ldots, u_{N}^{n}$, given by

$$
u_{i}^{n+1}=g_{i}\left(u_{0}^{n}, \ldots, u_{N}^{n}\right)=\left(e^{A k}\right)_{i} \boldsymbol{u}^{n}+k(\varphi(A, k))_{i} \boldsymbol{f}\left(\boldsymbol{\xi}, \boldsymbol{u}^{n}\right) .
$$

Assuming the boundedness of the derivative $\left|\frac{\partial F}{\partial u}\right| \leq \lambda, x \in \Omega, 0 \leq u \leq 1$, then from non-negativity of $e^{A k}$ and $\varphi(A, k)$ one gets

$$
\frac{\partial g_{i}}{\partial u_{j}^{n}} \geq\left(e^{A k}\right)_{i j}-k \lambda(\varphi(A, k))_{i j}, \quad 0 \leq i, j \leq N .
$$

Let us denote $\Psi(A, k)=e^{A k}-k \lambda \varphi(A, k)$, and the vector function

$$
g\left(u_{i}^{n}, \ldots, u_{N}^{n}\right)=\left[g_{1}, \ldots, g_{N}\right]^{T},
$$

then from (22) the Jacobian matrix $\frac{\partial g}{\partial u^{n}}$ satisfies

$$
\frac{\partial g}{\partial u^{n}} \geq \Psi(A, k) .
$$


Let us denote

$$
a_{0}=\min _{0 \leq j \leq N} a_{0}^{j}
$$

Note that the non-negativity of $\Psi(A, k)$ guarantees the non-negativity of $\frac{\partial g}{\partial u^{n}}$ and hence, $g_{i}$ increases in each direction $u_{j}^{n}$. In fact, from (8) and under condition (19), $B=A-a_{0} I$ verifies $B \geq 0$, and taking into account that $e^{A k}=e^{a_{0} k} e^{B k}, \Psi(A, k)$ can be written as follows

$$
\begin{gathered}
\Psi(A, k)=\phi_{0}(k) I+\sum_{s=1}^{\infty} \phi_{s}(k) \frac{B^{s} k^{s}}{s !}, \\
\phi_{0}(k)=e^{a_{0} k}-\frac{k \lambda}{6}\left(1+4 e^{a_{0} \frac{k}{2}}+e^{a_{0} k}\right), \\
\phi_{s}(k)=e^{a_{0} k}-\frac{k \lambda}{6}\left(\frac{4}{2^{s}} e^{a_{0} \frac{k}{2}}+e^{a_{0} k}\right) .
\end{gathered}
$$

Taylor expansion of (27) shows that for some $\xi$, such that $0<\xi<k$,

$$
\begin{aligned}
& \phi_{0}(k)=1-k\left(\lambda-a_{0}\right)+k^{2} \frac{\phi_{0}^{\prime \prime}(\xi)}{2} \\
& \phi_{0}^{\prime \prime}(\xi)=a_{0}^{2} e^{a_{0} \xi}+\frac{\lambda}{3}\left|a_{0}\right| e^{a_{0} \frac{\xi}{2}}+\frac{\lambda\left|a_{0}\right|}{6}\left(2-\left|a_{0}\right| \xi\right)\left(e^{a_{0} \xi}+e^{a_{0} \frac{\xi}{2}}\right) .
\end{aligned}
$$

Note that the sum of the two first terms of the Taylor expansion of $\phi_{0}(k)$, $1-k\left(\lambda-a_{0}\right)$ is positive, if $k<\frac{1}{\lambda+\left|a_{0}\right|}$, and by (8) this occurs when

$$
k<\frac{h^{2}}{2 d+\lambda h^{2}}, \quad d=\max _{\boldsymbol{x} \in \Omega} D(\boldsymbol{x}) .
$$

Condition (30) implies $2-\left|a_{0}\right| \xi>0$ and from $(29), \phi_{0}^{\prime \prime}(\xi)$ and $\phi_{0}(k)$ are positive. It is easy to check that for $s \geq 1, \phi_{s}(k) \geq \phi_{0}(k)>0$. Thus, Jacobian matrix $\frac{\partial g}{\partial u^{n}}$ is non-negative, and using induction hypothesis $u_{i}^{n} \leq 1$ and (21), non-negativity of $u_{i}^{n}$ and $F(x, 1)=0, x \in \Omega$, under conditions (19) and (30) for the interior points one gets

$$
0 \leq u_{i}^{n+1}=g_{i}\left(u_{0}^{n}, \ldots, u_{N}^{n}\right) \leq g_{i}(1, \ldots, 1)=\left\|\left(e^{A k}\right)_{i}\right\|_{\infty} \leq\left\|e^{A k}\right\|_{\infty}=1 .
$$

Summarizing all above, the main result of the paper is established as follows Theorem 3.1. With previous notation under conditions (19) and (30) the numerical solution $\boldsymbol{u}^{n}$ of the scheme (16) is non-negative and $\|\cdot\|_{\infty}$-stable, with ${ }_{90}\left\|\boldsymbol{u}^{n}\right\|_{\infty} \leq 1$ at any time level $0 \leq n \leq N_{t}$. 


\section{Applications and simulations}

In this section we present two multidimensional DAR problems to illustrate the proposed ETD technique.

\subsection{Fisher-Kolmogorov-Petrovsky-Piscounov equation}

A generalized diffusive logistic model finds an application in many fields. For two spatial dimensions it takes the form of the well known 2D FisherKolmogorov-Petrovsky-Piscounov equation [20, 21]

$$
\frac{\partial u}{\partial t}=a\left(\frac{\partial^{2} u}{\partial x^{2}}+\frac{\partial^{2} u}{\partial y^{2}}\right)+f(u)
$$

where the unknown $u(x, y, t)$ represents the distribution density of a species expressed as a function of the spatial coordinates $x, y$, and time $t$. Here $a>0$ is the diffusion coefficient, $f(u)=b u\left(1-u^{p}\right)$ is the source term with $b>0$ and $p \geq 1$. Without lost of generality, we take $a=b=1$. Following the method in [22], initial and boundary conditions are defined by a family of the travelling wave solutions with the arbitrary real numbers $\varphi$ and $C$ given in [23].

$$
\begin{aligned}
u^{ \pm}(x, y, t) & =\left[\frac{1}{2} \tanh \psi+\frac{1}{2}\right]^{2 / p}, \\
\psi & = \pm \frac{p}{2 \sqrt{2 p+4}}(x \sin \varphi+y \cos \varphi)+\frac{p(p+4)}{4(p+2)} t+C .
\end{aligned}
$$

Example 1. Let us consider Fisher-KPP equation with the parameters given in Table 1 for the cases a) and b) correspondingly, as it is proposed in [23]. In both cases the computational domain is a square symmetric with respect to $x$ and $y$ axes, such that $x_{\min }=y_{\min }=-x_{\max }=-y_{\max }$. The spatial discretization is presented by the uniform grid $h_{1}=h_{2}=h$.

The numerical solution for both examples is presented in Figures 1 and

2. The solid surface corresponds to the initial condition, the wireframe mesh correspond to the solution at the moment $t=T$. 


\begin{tabular}{|l|c|c|c|c|c|c|c|c|}
\hline & $p$ & $x_{\max }$ & $T$ & \pm & $C$ & $\varphi$ & $h$ & $k$ \\
\hline Example 1.a) & 1 & 20 & 10 & - & $-\ln \sqrt{10}$ & $\frac{3}{4} \pi$ & 1.00 & 0.01 \\
\hline Example 1.b) & 2 & 30 & 15 & + & $-\ln \sqrt{5}$ & $\frac{2}{3} \pi$ & 2.00 & 0.02 \\
\hline
\end{tabular}

Table 1: Parameters in Example 1.

\begin{tabular}{|c|ccc|}
\hline$h$ & \multicolumn{3}{|c|}{$k$} \\
& $10^{-1}$ & $10^{-2}$ & $10^{-4}$ \\
\hline 4 & $2.8985 \mathrm{e}-03$ & $1.2824 \mathrm{e}-03$ & $1.1425 \mathrm{e}-03$ \\
2 & $2.4343 \mathrm{e}-03$ & $4.8579 \mathrm{e}-04$ & $3.2553 \mathrm{e}-04$ \\
1 & $2.3595 \mathrm{e}-03$ & $2.8714 \mathrm{e}-04$ & $8.5989 \mathrm{e}-05$ \\
0.5 & $2.3206 \mathrm{e}-03$ & $2.5000 \mathrm{e}-04$ & $2.2995 \mathrm{e}-05$ \\
\hline
\end{tabular}

Table 2: Relative errors of the numerical solution with respect to the exact solution at $t=1$ in Example 1.a).

The relative error at $t=1$ for the numerical solution by the proposed ETD method in Example 1.a) is presented in Table 2. Results demonstrate competitive accuracy with respect to the semi-explicit finite difference methods proposed in [23].

\subsection{Multi-asset American basket option}

The considered approach can be applied to multi-dimensional Black-Scholes (BS) equation. Let $S_{1}, \ldots, S_{M}$ be the asset prices, where $M$ is the number of assets in a portfolio. Let us denote the vector of asset prices $\mathbf{S}=\left(S_{1}, \ldots, S_{M}\right)^{T}$ and $P(\mathbf{S}, \tau)$ be the value of American basket option at the moment $\tau$, where $\tau$ is time to maturity $T$, with the payoff function

$$
P(\mathbf{S}, 0)=\left(E-\sum_{i=1}^{M} \alpha_{i} S_{i}\right)^{+},
$$

where $E$ is the strike price and $\alpha_{i}$ is the positive weight of the corresponding $i$-th asset in the basket. Assuming that the asset prices follow a geometric Brownian 

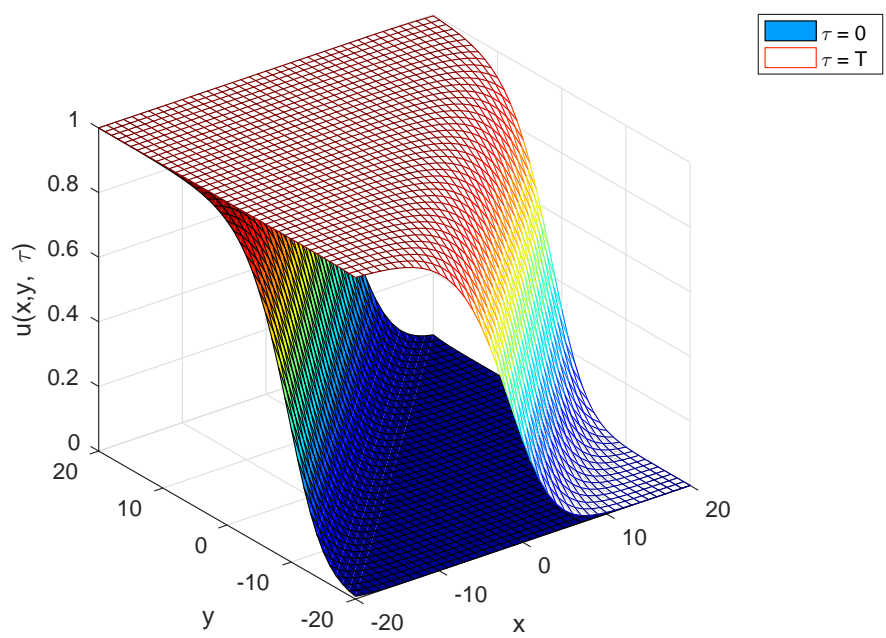

Figure 1: Example 1.a).

motion, using Martingale strategies, no-arbitrage principle and Itô's calculus

$$
\begin{aligned}
\frac{\partial P}{\partial \tau}= & \frac{1}{2} \sum_{i=1, j=1}^{M} \rho_{i j} \sigma_{i} \sigma_{j} S_{i} S_{j} \frac{\partial^{2} P}{\partial S_{i} \partial S_{j}}+\sum_{i=1}^{M}\left(r-q_{i}\right) S_{i} \frac{\partial P}{\partial S_{i}}-r P+F(P), \\
S_{i} & >0, \quad i=1, \ldots, M, \quad 0<\tau \leq T
\end{aligned}
$$

where $\sigma_{i}$ is the volatility of $S_{i}, \rho_{i j}$ is the correlation between $S_{i}$ and $S_{j}, r$ is the risk-free rate, $q_{i}$ is the constant dividend yield of $i$-th asset.

Let us denote matrix $R \in \mathbb{R}^{M \times M}$ as the correlation matrix with entries $\rho_{i j}$, satisfying $-1 \leq \rho_{i j} \leq 1$. The nonlinear penalty term $F(P)$ has several suitable forms $[25,26]$. Here we chose the following type, see [25],

$$
F(P)=\lambda(P(\mathbf{S}, 0)-P(\mathbf{S}, \tau))^{+},
$$

where $\lambda$ is non-negative. This penalty term is in accordance with recent rationality parameter approach $[27,28]$, that takes into account that the buyer does not exercise when it is not profitable. 

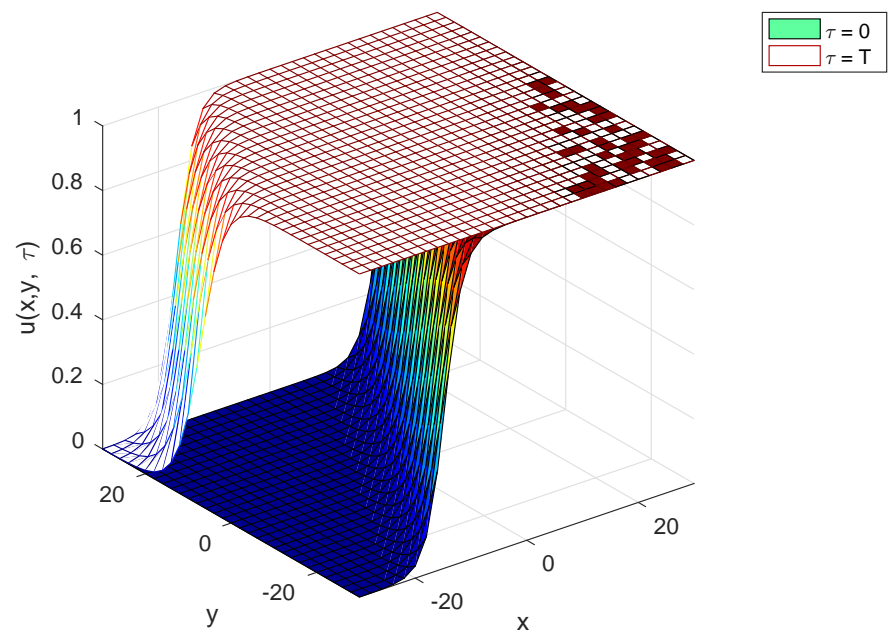

Figure 2: Example 1.b) .

Note that at each boundary $S_{i}=0$ the Black-Scholes equation for $M-1$ assets is established and

$$
\lim _{S_{i} \rightarrow \infty} P\left(S_{1}, \ldots, S_{i}, \ldots, \tau\right)=0, \quad 1 \leq i \leq M .
$$

In the case of two underlying assets after the cross-derivative elimination proposed in [29] and following notation therein, (36) takes the DAR problem form

$$
\begin{aligned}
\frac{\partial U}{\partial \tau} & =\frac{1}{2} \frac{\partial^{2} U}{\partial y_{1}^{2}}+\frac{1}{2}\left(1-\rho^{2}\right) \frac{\partial^{2} U}{\partial y_{2}^{2}}+\delta_{1} \frac{\partial U}{\partial y_{1}} \\
& +\left(\delta_{2}-\rho \delta_{1}\right) \frac{\partial U}{\partial y_{2}}-r U+\lambda(U(\mathbf{y}, 0)-U(\mathbf{y}, \tau))^{+},
\end{aligned}
$$

where $\left(y_{1}, y_{2}\right) \in \mathbb{R}^{2}, 0<\tau \leq T$, and

$$
y_{1}=\frac{1}{\sigma_{1}} \ln \frac{S_{1}}{E}, y_{2}=\frac{1}{\sigma_{2}} \ln \frac{S_{2}}{E}-\frac{\rho}{\sigma_{1}} \ln \frac{S_{1}}{E}, U\left(y_{1}, y_{2}, \tau\right)=\frac{1}{E} P\left(S_{1}, S_{2}, \tau\right) .
$$


Initial condition takes the following form

$$
U\left(y_{1}, y_{2}, 0\right)=\left(1-\alpha_{1} e^{\sigma_{1} y_{1}}-\alpha_{2} e^{\sigma_{2}\left(y_{2}+\rho y_{1}\right)}\right)^{+} .
$$

Numerical solution is found in bounded domain $\left[y_{1_{\min }}, y_{1_{\max }}\right] \times\left[y_{2_{\min }}, y_{2_{\max }}\right]$, such that boundary conditions are fulfilled.

Then the semi-discretized in space approximation of (39) takes the following five-point stencil form

$$
\begin{aligned}
\frac{d u_{i, j}}{d \tau} & =a_{-2} u_{i, j-1}+a_{-1} u_{i-1, j}+a_{0} u_{i, j}+a_{1} u_{i+1, j}+a_{2} u_{i, j+1} \\
& +\lambda\left(u_{i, j}(0)-u_{i, j}(\tau)\right)^{+}
\end{aligned}
$$

$$
\begin{gathered}
a_{0}=-\frac{1}{h^{2}}\left(\frac{1}{\beta_{1}^{2}}+\frac{1-\rho^{2}}{\beta_{2}^{2}}+r h^{2}\right), \quad a_{ \pm 1}=\frac{1}{h^{2}}\left(\frac{1}{2 \beta_{1}^{2}} \pm \frac{h \delta_{1}}{2 \beta_{1}}\right), \\
a_{ \pm 2}=\frac{1}{h^{2}}\left(\frac{1-\rho^{2}}{2 \beta_{2}^{2}} \pm \frac{h}{2 \beta_{2}}\left(\delta_{2}-\rho \delta_{1}\right)\right) .
\end{gathered}
$$

In this case we include the linear reaction term $-r u$ into the homogeneous part $A \mathbf{u}$ of equation (11), because the matrix $A$ remains a Metzler one and the nonlinear source term is non-negative. Theorem 3.1 is applied to (42), the nonlinear source term (37) admitting piecewise bounded derivative with respect to $P$, or $U$ after the transformation. In this case stability condition (30) takes the form

$$
k<\frac{h^{2}}{d+(r+\lambda) h^{2}}, \quad d=\frac{1}{\beta_{1}^{2}}+\frac{1-\rho^{2}}{\beta_{2}^{2}} .
$$

Next, we present some numerical results. Example 2 shows that the stability condition (45) is crucial: if the condition is broken, the numerical results can be wrong. The implementation of the proposed method has been done by using MatLAB R2015a on processor Pentium(R) Dual-Core CPU E5700 3.00 GHz. The results of the following examples are presented in original variables $(\mathbf{S}, \tau)$ obtained by the inverse transformation. 
Example 2. We consider American basket put option with no dividends payments pricing with the following parameters

$$
\sigma_{1}=0.65, \sigma_{2}=0.25, r=0.05, \rho=0.1, \alpha_{1}=\alpha_{2}=0.5, T=1, E=9 .
$$

The penalty parameter is chosen $\lambda=100, \beta_{1}=\beta_{2}=1$. Transformed computational domain is $[-8,8] \times[-8,8]$. In Figures 3 and 4 the option price is presented for various $h$ and according to (45), fixed $k=8 \cdot 10^{-3}$. If time step is chosen larger, for example, $k=0.05$ or $k=0.1$ (see Figures 5 and 6 ), the solution exceeds the strike value $E$, which does not agree with reality.

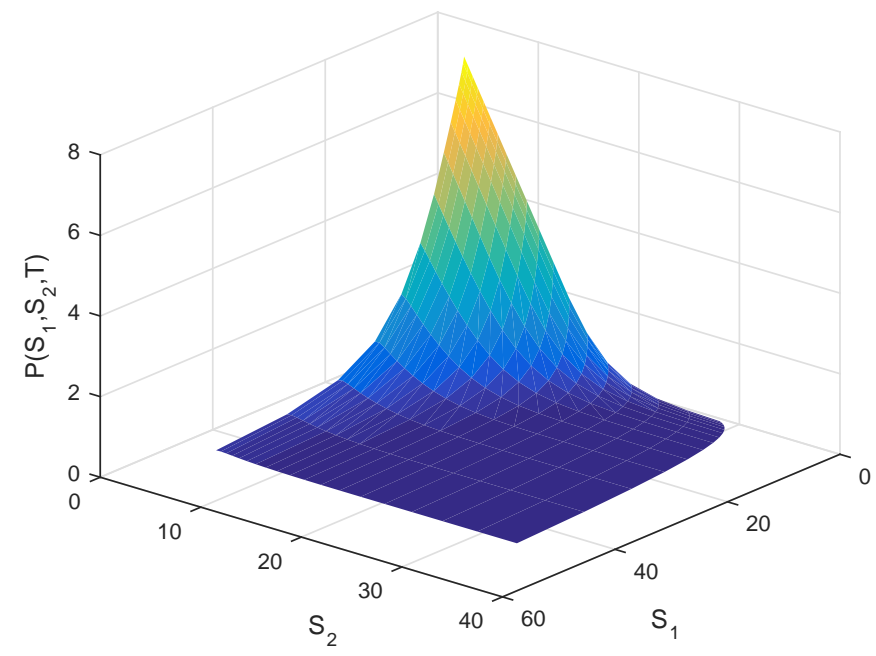

Figure 3: Reliable basket option price of Example 2 at $\tau=T$ for $h=0.5$.

Example 3 deals with comparison between the proposed scheme and the tree method of [30]. Influence of the parameter $\lambda$ to the solution is also studied.

Example 3. The American basket put option of two assets is considered with the following parameters [30]

$$
\sigma_{1}=0.3, \sigma_{2}=0.2, r=0.05, \rho=0.6, \alpha_{1}=0.7, \alpha_{2}=0.3, T=1, E=50 .
$$




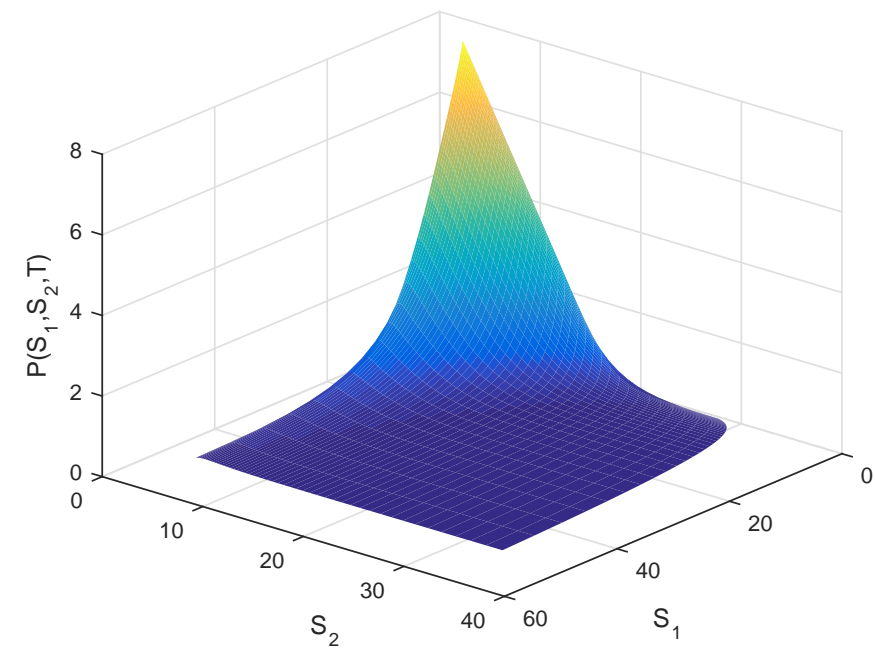

Figure 4: Reliable basket option price of Example 2 at $\tau=T$ for $h=0.2$.

As a reference value at the point $\mathbf{S}=(50,50)$ the result of the Binomial Tree method of [30] is used. The results of the proposed method with various spatial stepsizes $h$ and fixed $k=5 \cdot 10^{-3}$, in the computational spatial domain $[-8,8] \times[-8,8]$ are presented in Table 3 .

\begin{tabular}{|c|c|cc|}
\hline$h$ & Number of nodes & Value & Ratio \\
\hline 0.8 & $21 \times 21$ & 3.7075 \\
0.4 & $41 \times 41$ & 3.9537 & 12.5047 \\
0.2 & $81 \times 81$ & 3.9730 & 10.1905 \\
0.1 & $161 \times 161$ & 3.9747 & 5.2500 \\
\hline \multicolumn{2}{|c|}{ Tree method $(P)$} & \multicolumn{2}{|c|}{3.9751} \\
\hline
\end{tabular}

Table 3: Comparison of option price for Example 3.

The convergence ratio is the factor by which the error decreases at each grid refinement. It is presented in Table 3, where the absolute error is computed as 


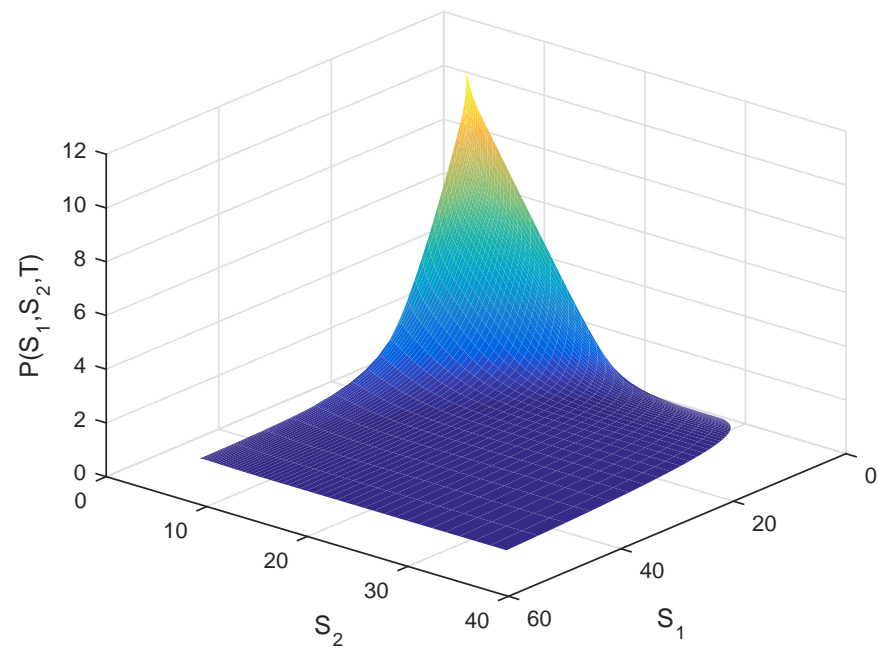

Figure 5: Wrong basket option price of Example 2 at $\tau=T$ with broken stability conditions $(k=0.05, h=0.2)$.

follows

$$
\epsilon_{h}=\left|P_{h}-P\right|,
$$

where $P_{h}$ is the computed value of the option, $P$ is the reference value obtained

by the tree method in [30]. The error $\epsilon$ is plotted for various stepsizes $h$ in Figure 7.

The choice of timestep $k$ depends on the value of the parameter $\lambda$. In Table 4 values of the basket option with parameters $(47)$ at $\mathbf{S}=(50,50)$ applying fixed spatial stepsize $h=0.2$ are presented depending on $\lambda$.

The numerical simulations of Example 3 show that the accuracy remains almost fixed for values of $\lambda>100$. It is advisable to chose $\lambda$ about 100 to save the computational time.

The proposed method can be applied not only for put options, but also for 


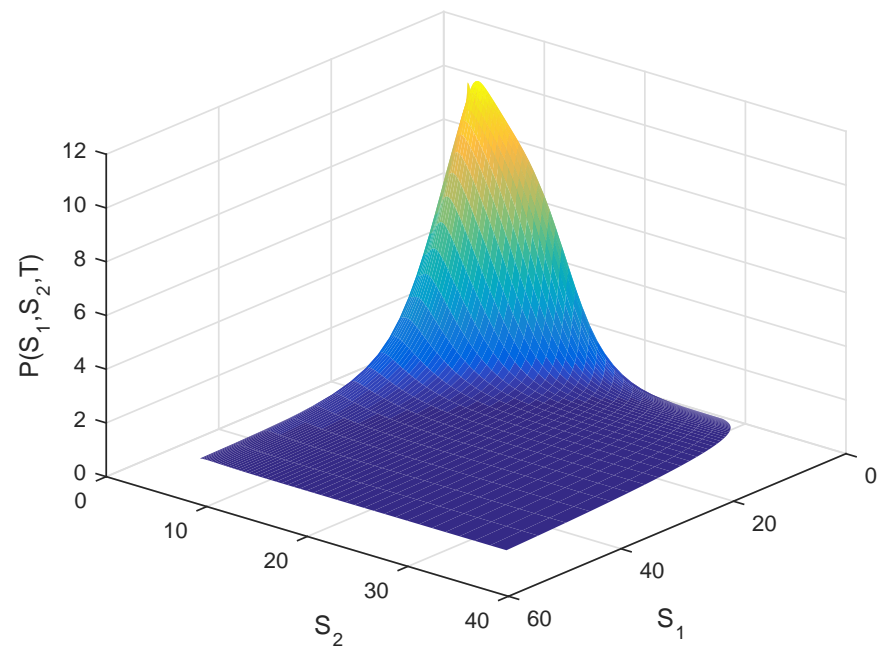

Figure 6: Wrong basket option price of Example 2 at $\tau=T$ with broken stability conditions $(k=0.1, h=0.2)$.

call options. Initial condition in this case takes the following form

$$
P(\mathbf{S}, 0)=\left(\sum_{i=1}^{M} \alpha_{i} S_{i}-E\right)^{+} .
$$

Example 4 provides numerical solution for American basket call option and its comparison with high order finite element method of [15].

Example 4. The American basket call option of two assets with the strike price $E=100 \$$ is considered with the following parameters [15]

$$
\sigma_{1}=0.12, \sigma_{2}=0.14, r=0.03, \rho=0.3, q_{1}=0.01, q_{2}=0.01, T=0.5 .
$$

In Table 5 we include the results at $\mathbf{S}=(100,100)$ for $\lambda=100$, various spatial stepsizes $h$ and corresponding $k$ under condition (30). The numerical solution by high-order computational method of [15] is denoted by HOC. The numerical solution at $\tau=T$ and the payoff for American basket call options are presented in Figures 8 and 9. 


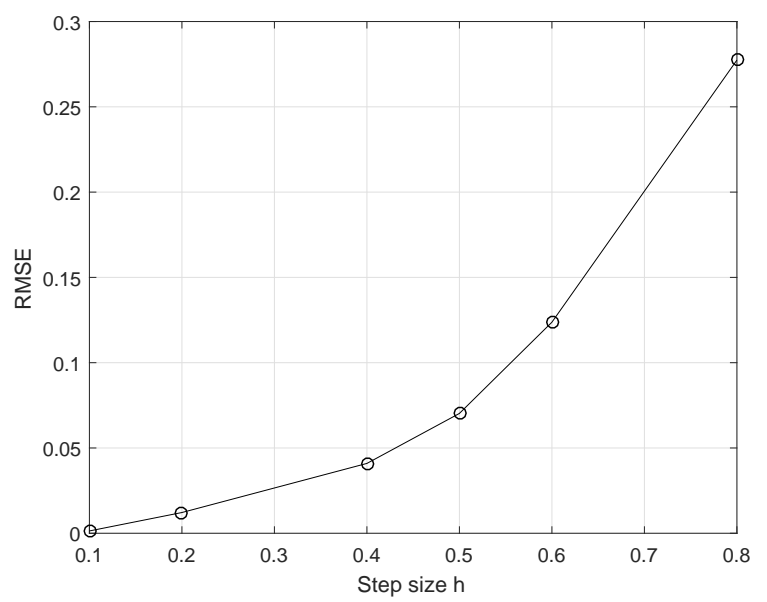

Figure 7: Absolute error $\epsilon_{h}$ of the proposed method in Example 2 for various $h$.

180

\section{Conclusions}

In this paper multidimensional DAR problems are solved by a proposed ETD method paying a special attention to the numerical analysis of the fully discretized scheme instead of the stability of the solution of the system of ODEs freezing spatial step sizes.

185

In fact, using logarithmic norm of matrices and properties of matrix exponential, sufficient condition on the step sizes are given, so that the numerical solution of the difference scheme remains norm bounded as the stepsizes tend to zero and the size of the involved matrices grows infinitely. Moreover, these conditions are sufficient for the positivity of the solution, that is important dealing with real physical objects, as concentrations, populations, prices, etc.

\section{Acknowledgements}

This work has been partially supported by the Ministerio de Economía y Competitividad Spanish grant MTM2017-89664-P. 


\begin{tabular}{|c|c|}
\hline$\lambda$ & $P_{h}$ \\
\hline 0 & 3.6583 \\
1 & 3.7869 \\
10 & 3.9288 \\
100 & 3.9730 \\
1000 & 3.9732 \\
10000 & 3.9733 \\
\hline Tree method $(P)$ & 3.9751 \\
\hline
\end{tabular}

Table 4: Option price for the parameters (47).

\begin{tabular}{|c|c|c|}
\hline Nodes & Proposed method & HOC \\
\hline $12 \times 12$ & 3.18982 & 2.86247 \\
$24 \times 24$ & 3.35338 & 3.27894 \\
$48 \times 48$ & 3.41344 & 3.35094 \\
\hline
\end{tabular}

Table 5: American basket call option price comparison for Example 4.

\section{References}

[3] F. Yi, J. Wei, J. Shi, Bifurcation and spatiotemporal patterns in a homogeneous diffusive predatorprey system, Journal of Differential Equations 246 (5) (2009) $1944-1977$.

[4] J. Hull, Options, Futures and Other Derivatives, Pearson/Prentice Hall, 2009. 


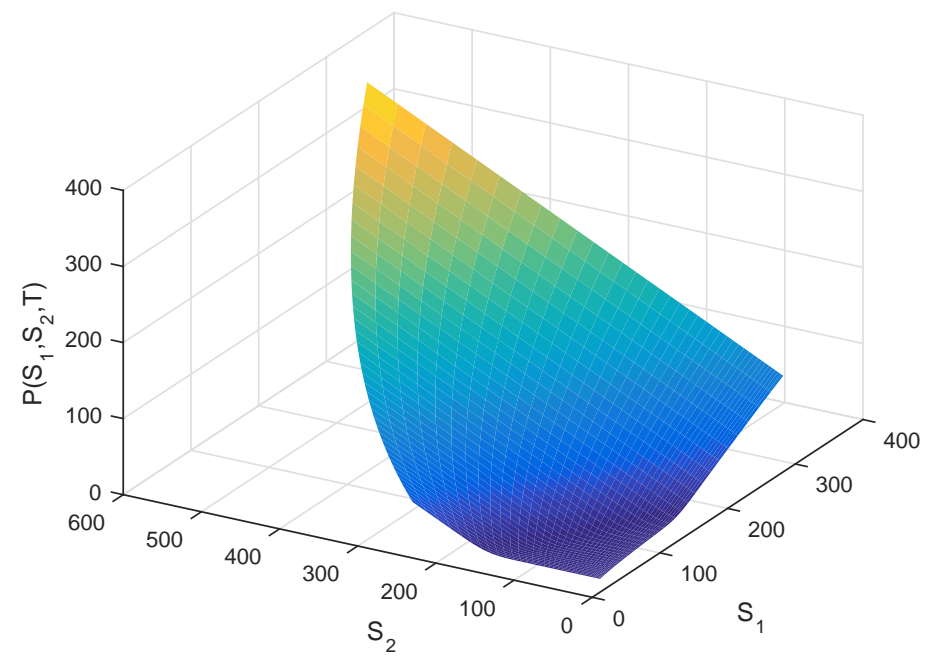

Figure 8: Basket call option price with parameters (50) at $\tau=T$.

[5] D. A. Garzón-Alvarado, C. Galeano, J. Mantilla, Computational examples of reaction-convection-diffusion equations solution under the influence of fluid flow: First example, Applied Mathematical Modelling 36 (10) (2012) $5029-5045$.

[6] B. Gustafsson, H. O. Kreiss, J. Oliger, Time-Dependent Problems and Difference Methods, John Wiley \& Sons, Inc., 1995.

[7] M. G. Crandall, H. Ishii, P.-L. Lions, User's guide to viscosity solutions of second order partial differential equations, Bulletin of the American Mathematical Society 27 (1992) 1-67.

[8] E. Cokca, A computer program for the analysis of 1-d contaminant migration through a soil layer, Environmental Modelling \& Software 18 (2) (2003) $147-153$.

[9] M. Calvo, J. de Frutos, J. Novo, Linearly implicit Runge-Kutta methods 


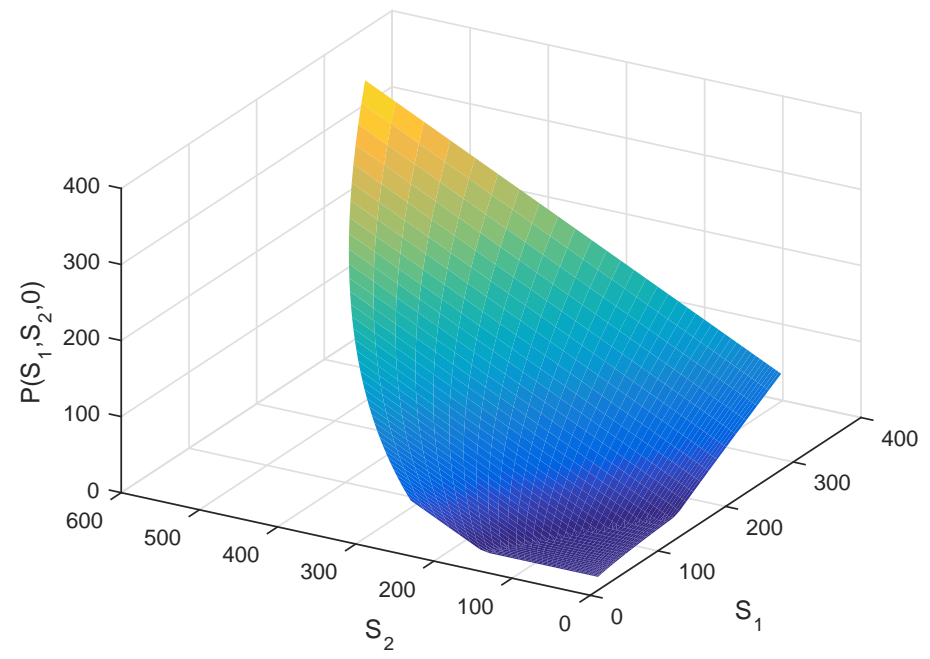

Figure 9: Basket call option price with parameters (50) at $\tau=0$.

for advection-reaction-diffusion equations, Applied Numerical Mathematics 37 (4) (2001) $535-549$.

220 [10] J. Macías-Díaz, A. Puri, An explicit positivity-preserving finite-difference scheme for the classical Fisher-Kolmogorov-Petrovsky-Piscounov equation, Applied Mathematics and Computation 218 (9) (2012) 5829 - 5837.

[11] A. Kaya, Finite difference approximations of multidimensional unsteady convectiondiffusionreaction equations, Journal of Computational Physics 285 (2015) 331-349.

[12] S. Cox, P. Matthews, Exponential time differencing for stiff systems, Journal of Computational Physics 176 (2) (2002) 430 - 455.

[13] F. de la Hoz, F. Vadillo, Numerical simulations of time-dependent partial differential equations, Journal of Computational and Applied Mathematics 295 (2016) $175-184$. 
[14] A.-K. Kassam, L. N. Trefethen, Fourth-order time-stepping for stiff pdes, SIAM Journal on Scientific Computing 26 (4) (2005) 1214-1233.

[15] N. Rambeerich, D. Tangman, M. Lollchund, M. Bhuruth, High-order computational methods for option valuation under multifactor models, European Journal of Operational Research 224 (1) (2013) 219 - 226.

[16] K. E. Atkinson, An Introduction to Numerical Analysis, John Wiley \& Sons, Inc., 1989.

[17] T. Kaczorek, Positive 1D and 2D Systems, Springer London, 2002.

[18] G. Dahlquist, Stability and error bounds in the numerical integration of ordinary differential equations, Almqvist \& Wiksells, Uppsala, 1958; Transactions of the Royal Institute of Technology, Stockholm.

[19] C. A. Desoer, M. Vidyasagar, Feedback Systems: Input-Output Properties, Academic Press, 1975.

[20] R. A. Fisher, The wave of advance of advantageous genes, Annals of Eugenics 7 (4) (1937) 355-369.

[21] A. Kolmogorov, N. Petrovsky, S. Piscounov, tude de lquations de la diffusion avec croissance de la quantit de matire et son application a un problme biologique, Bull. Univ. Moskou 1 (1937) 1-25.

[22] P. K. Brazhnik, J. J. Tyson, On traveling wave solutions of Fisher's equation in two spatial dimensions, SIAM Journal on Applied Mathematics 60 (2) (1999) 371-391.

[23] W. Qin, D. Ding, X. Ding, Two boundedness and monotonicity preserving methods for a generalized Fisher-KPP equation, Applied Mathematics and Computation 252 (2015) 552 - 567.

[24] D. Tavella, C. Randall, Pricing Financial Instruments: The Finite Difference Method, John Wiley and Sons, New York, 2007. 
[25] P. A. Forsyth, K. R. Vetzal, Quadratic convergence for valuing american options using a penalty method, SIAM Journal of Scientific Computing 23 (2002) 2095-2122.

[26] B. F. Nielsen, O. Skavhaug, A. Tveito, Penalty methods for the numerical solution of American multi-asset option problems, Journal of Computational and Applied Mathematics 222 (1) (2008) 3 - 16, special Issue: Numerical $\{\mathrm{PDE}\}$ Methods in Finance.

[27] K. S. T. Gad, J. L. Pedersen, Rationality parameter for exercising American put, Risks 3 (2) (2015) 103.

[28] R. Company, V. Egorova, L. Jódar, C. Vázquez, Finite difference methods for pricing american put option with rationality parameter: Numerical analysis and computing, Journal of Computational and Applied Mathematics 304 (2016) 1-17.

[29] R. Company, V. Egorova, L. Jódar, F. Soleymani, A mixed derivative terms removing method in multi-asset option pricing problems, Applied Mathematics Letters 60 (2016) 108 - 114.

[30] S. Borovkova, F. Permana, J. van der Weide, American basket and spread option pricing by a simple binomial tree, The Journal of Derivatives Summer (2012) 29-38. 